\title{
Editorial. Confinement des étrangers : entre circulation et enfermement
}

Editorial. Alien exclusion: between circulation and confinement

Editorial. El confinamiento de los extranjeros: entre la circulación y la reclusión

\section{Chowra Makaremi et Carolina Kobelinsky}

\section{(2) OpenEdition}

\section{Journals}

\section{Édition électronique}

URL : http://journals.openedition.org/conflits/15943

DOI : $10.4000 /$ conflits. 15943

ISSN : $1777-5345$

Éditeur :

CCLS - Centre d'études sur les conflits lilberté et sécurité, L'Harmattan

Édition imprimée

Date de publication : 30 octobre 2008

Pagination : 7-11

ISBN : 978-2-296-06624-3

ISSN : 1157-996X

Référence électronique

Chowra Makaremi et Carolina Kobelinsky, «Editorial. Confinement des étrangers : entre circulation et enfermement », Cultures \& Conflits [En ligne], 71 | automne 2008, mis en ligne le 02 février 2009, consulté le 30 mars 2021. URL : http://journals.openedition.org/conflits/15943 ; DOI : https://doi.org/ 10.4000/conflits. 15943 


\title{
Editorial. Confinement des étrangers : entre circulation et enfermement ${ }_{1}$
}

\author{
Chowra MAKAREMI
}

Chowra Makaremi est doctorante en anthropologie à l'université de Montréal. Ses recherches portent sur la détention frontalière en France. Elle a notamment publié "Alien Confinement in Europe: Violence and the Law. The Case of Roissy-Charles de Gaulle Airport in France", in Hogan C., Marín Dòmine M., The Camp: Narratives of Internment and Exclusion, Newcastle, Cambridge Scholars Publishing, 2007 (pp. 39-54).

\section{Carolina KOBELINSKY}

Carolina Kobelinsky est doctorante en anthropologie à l'EHESS, rattachée à l'Institut de recherche interdisciplinaire sur les enjeux sociaux. Ses recherches portent sur la politique d'asile en France. Elle a récemment publié "The Moral Judgment of Asylum Seekers in French Reception Centers", Focus commentary, Anthropology News, mai 2008 (pp. 5-11).

«C'est vers l'autre monde que part le fou sur sa folle nacelle ; c'est de l'autre monde qu'il vient quand il débarque. [...] Situation symbolique et réalisée à la fois par le privilège qui est donné au fou d'être enfermé aux portes de la ville : son exclusion doit l'enclore; s'il ne peut et ne doit voir d'autre prison que le seuil luimême, on le retient sur le lieu du passage. Il est mis à l'extérieur de l'intérieur, et inversement. [...] Enfermé

1. Ce numéro découle d'une conférence pluridisciplinaire sur «Le confinement des étrangers en Europe : perspectives de terrain ", qui s'est déroulée les 3 et 4 septembre 2007 à l'Ecole des hautes études en sciences sociales à Paris, dans le cadre du programme ANR «Au-delà de la "question immigrée”. Les nouvelles frontières de la société française» et avec le soutien du réseau TERRA. Chercheurs et doctorants de plusieurs pays européens, politistes, sociologues, juristes, anthropologues et philosophes ont été réunis par cette initiative, dont l'objectif était moins d'ériger un tableau comparatif des espaces de confinement des étrangers en Europe que de nourrir la réflexion d'un regard croisé sur différents contextes nationaux de recherche, à partir de diverses approches disciplinaires. 
dans le navire, d'où il n'échappe pas, le fou est confié à la rivière aux mille bras, à la mer aux mille chemins, à cette grande incertitude extérieure à tout. Il est prisonnier au milieu de la plus libre, de la plus ouverte des routes : solidement enchaîné à l'infini carrefour. Il est le Passager par excellence, c'est-à-dire le prisonnier du passage. Et la terre sur laquelle il abordera, on ne la connait pas, tout comme on ne sait pas, quand il prend pied, de quelle terre il vient. Il n'a sa vérité et sa patrie que dans cette étendue inféconde entre deux terres qui ne peuvent lui appartenir.»

Foucault M., Histoire de la folie à l'âge classique ${ }^{2}$

\begin{abstract}
Le Passager par excellence, c'est-à-dire le prisonnier du passage » : c'est de « ce paradoxe que nous aimerions enrichir la notion de « mise à l'écart » développée ces dernières années pour qualifier les politiques et les pratiques de restrictions migratoires, qui engagent une réflexion autour de l'idée de clôture enfermer des personnes et fermer des frontières. Tandis que se désactivent les frontières pour la circulation des marchandises, des capitaux (et des personnes dans certaines zones de libre circulation), s'opèrent un renforcement des frontières et une intensification du marquage symbolique contre la circulation des personnes venant du Sud. Les pratiques de détention et d'expulsion des étrangers au sein des Etats occidentaux renvoient en effet à des processus d'entrave institutionnalisée de la circulation et à des dispositifs de confinement, qui reconfigurent ensemble la question politique de la gestion migratoire. Or, les enjeux de détention et d'expulsion ont besoin d'être réinscrits dans un espace de circulation ambigu, cartographié par une constellation de lieux disciplinaires, de poches d'attente, de pratiques de recensement et d'assistance. C'est dans cette actualité que s'inscrit le présent numéro, qui regroupe une série d'études empiriques sur les acteurs et les technologies du confinement des étrangers. Ces analyses cherchent à mettre l'accent sur la circulation des personnes et les contraintes qui en définissent les trajectoires, en s'attachant aux processus de régulation et de contrôle dans lesquelles celle-ci s'agencent.
\end{abstract}

Nous souhaitons montrer comment, concrètement, la « mise à l'écart » procède tout aussi bien d'une accumulation de situations d'enfermement que d'une constellation de lieux, qui sont en même temps des espaces transitoires de la circulation. Ces espaces dessinent les nœuds d'un parcours marqué par des déambulations forcées, des traversées de frontières, qui peuvent se désactiver et se réactiver, se durcir ou s'assouplir. Cette dimension supplémentaire, qui est celle du mouvement, implique ainsi une approche dynamique du confinement, non plus seulement en termes d' " enfermement dans ", mais également en termes de

2. Foucault, M., Histoire de la folie à l'âge classique, Paris, Gallimard, 1972, p. 22. 
déplacements au sein d'un espace parallèle, à la marge. Non pas que la réalité de l'enfermement des étrangers ait profondément changé, mais aux côtés d'un regard sur ces questions - la criminalisation, la privation de liberté et l'existence de poches d'arbitraires -, il s'agit d'élargir la perspective afin de comprendre le camp ${ }^{3}$ dans son rapport complexe aux pratiques de contrôle de la circulation et aux espaces de circulation contraints ou illégaux qu'il produit en creux. Le camp est un enfermement, mais aussi un transit qui permet d'éclairer le régime de contrôle de la circulation fait de nœuds et de circulation limbaire, de détention et de mise sur orbite. Que ce soit dans les postes de police de l'aéroport de Roissy-Charles de Gaulle qu'observe Morgane Iserte, dans les zones d'attente évoquées par Christel Cournil et Chowra Makaremi, ou dans les Centres d'accueil pour demandeurs d'asile (CADA) étudiés par Carolina Kobelinsky, le quotidien des pratiques de confinement renvoie à quelque chose qui les déborde et qui se lit en termes de parcours, de va-et-vient dans et hors la légalité, de construction subjective autour de rapports de force ambigus, parce qu'ils impliquent autant la prise en charge que le contrôle et la coercition. Ainsi, la réflexion sur l'enfermement doit entrer en dialogue et se confronter à une réflexion sur le contrôle, où les rapports de force s'extraient des thèmes de privation de liberté propres au disciplinaire pour se réinscrire dans des flux, que l'on trie ou filtre, et des mouvements, que l'on gère ${ }^{4}$.

Dans cette perspective, le contexte européen met en place un espace stratifié et complexe qui institue les acteurs de la circulation dans un quadrillage de pratiques et de normes fait de superpositions, de différences nationales - comme les législations sur le travail des demandeurs d'asile et sur le regroupement familial - et d'homogénéisations, comme les accords de Dublin II et ceux de Schengen. Mathilde Darley souligne ces tensions à travers les politiques nationales et les pratiques de coopération du contrôle frontalier tchéco-autrichien, en étudiant cette frontière - alors extérieure - de l'Union européenne et son intégration au sein de l'espace Schengen à partir de 2007. C'est dans ce contexte que s'inscrit la directive européenne "retour ", censée harmoniser les législations relatives à la rétention et l'expulsion des étrangers en situation irrégulière. Adoptée par le Parlement européen le 18 juin 2008, ce texte prévoit une possibilité d'enfermement allant jusqu'à 18 mois (alors qu'en France, la durée maximale de rétention était de 32 jours) afin de préparer l'éloignement des étrangers. Le texte prévoit notamment la détention et le renvoi des femmes enceintes, des personnes âgées et des mineurs qu'ils soient accompagnés ou non, inscrivant une avancée significative du contrôle dans le jeu des pratiques administratives, des résistances du droit et des processus législatifs, qu'analyse Christel Cournil. Son article propose ainsi une lecture juridique pertinente pour mettre en perspective ces dernières évolutions et les jeux de pouvoirs dans lesquels elles s'inscrivent.

3. Bernardot M., Camps d'étrangers, Bellecombre-en-Bauges, Editions du Croquant, 2008.

4. Deleuze G., «Post-Scriptum sur les sociétés de contrôle », Pourparlers (1972-1990), Paris, Minuit, 1990. 
Cette directive, incarnant l'actualité d'une extrême précarisation du droit des étrangers et de son instrumentalisation politique, rappelle la nécessité d'examiner les évolutions de la gestion des populations aux frontières dans le cadre de la construction européenne, qu'abordaient déjà les numéros de Cultures E Conflits consacrés aux logiques et aux effets du visa Schengen ( $n^{\circ} 49$ et $\left.n^{\circ} 50\right)$. Ce numéro prolonge la réflexion et cherche à en explorer de nouveaux aspects en croisant les perspectives disciplinaires. L'article de Morgane Iserte étudie ainsi les technologies d'identification, de surveillance et de traçabilité qui reconfigurent les frontières de l'espace Schengen dans l'aéroport de Roissy. Une autre dimension qui s'ajoute à ce débat est celle du recours à une juridiction supranationale, la Cour européenne des droits de l'Homme (CEDH), dans les tentatives d'encadrement des pratiques régaliennes de contrôle, ce à quoi s'intéresse Christel Cournil en analysant l'évolution du droit des étrangers et les réponses législatives nationales au regard de la jurisprudence européenne.

Comment les dispositifs d'enfermement s'instituent-ils dans un contexte et une tradition nationale singuliers, tout en engageant des logiques transnationales, des enjeux globaux, des formes politiques nouvelles ? Le dispositif de contrôle pose la gestion administrative de l'enfermement dans une certaine économie nationale qui appelle, d'une part, la question de l'exercice du pouvoir étatique dans ces marges où Etat et société ne se rabattent pas l'un sur l'autre, et, d'autre part, la question de la relation du national à l'étranger, telle que posée par Sarah S. Willen dans le contexte israélien. Les pratiques de confinement et d'expulsion forcés s'inscrivent en effet dans un réseau d'espaces intermédiaires qui sont des espaces de confinement, de « hors-lieux 5 » du pouvoir étatique, mais où la question de la souveraineté n'est pas évacuée et se pose, au contraire, de façon renouvelée. Ce qui reste dehors, dans les « hors-lieux » de nos démocraties, n'est pas défini une fois pour toutes : il ne préexiste pas au processus d'exclusion. Le dispositif sécuritaire tend au contraire à le faire indéfiniment advenir ${ }^{6}$, dans le travail d'une frontière mouvante et réversible. La norme qui délimite ce dehors n'est donc pas définie au préalable : c'est la frontière qui la fait exister à travers des techniques et des pratiques de gouvernement des corps. L'enfermement dans la circulation touche un point sensible de la gouvernementalité, dans la mesure où l'on se propose d'entrer dans les paradoxes et la violence d'une pratique de régulation différentielle, en la montrant dans l'exercice le plus fin d'une gestion des corps à « faire circuler».

Cette gestion procède de qualifications administratives et juridiques. Ces catégorisations multiples et parfois concurrentes - sur lesquelles se penche l'article de Chowra Makaremi - sont le lieu où les normes du droit qui encadrent

5. Brossat A., "Zones d'attente, centre de rétention et "libertés" policières ", in Le Cour Grandmaison O., Lhulier G. et Valluy J. (dir.), Le Retour des camps? Sangatte, Lampedusa, Guantanamo..., Paris, Autrement, 2007.

6. Foucault M., Il faut défendre la société, Paris, Gallimard, 1997. 
en général les pratiques étatiques, et en particulier le traitement des étrangers, se confrontent à des mécanismes qui «jouent dans l'interstice des lois, selon des modalités bétérogènes au droit 7 » et en soumettant l'enjeu de légalité à des impératifs politiques de régularité et d'ordre. Or, ces catégorisations sont aussi porteuses d'effets de subjectivation : elles opèrent une "réduction des trajectoires individuelles, des individus, des hommes, des femmes, en corps : corps indistincts, corps déplacés, corps localisés qui se trouvent dès lors catalogués et définis comme réfugiés, immigrants, clandestins... ${ }^{8}$ ». Les contributions et les situations sur lesquelles ce numéro se penche ont en effet un horizon en commun, celui de l'expulsion des étrangers indésirables. Parler d'enfermement à travers la circulation revient ainsi à restituer cette pratique dans un réseau dont l'objet ultime est de faire bouger des corps à travers des frontières. Quels sont les modes de vie juridiques qui accompagnent et réalisent ce contrôle ? Comment prise en charge, tri et expulsion des corps s'articulent-ils ?

C'est dans cet esprit que les contributions de ce numéro explorent la tension et l'oscillation entre circulation et enfermement. Les deux premières s'attachent aux dispositifs aéroportuaires et administratifs de contrôle de la circulation. Mathilde Darley présente une ethnographie des pratiques locales de contrôle déployées à la frontière tchéco-autrichienne entre 2004 et 2007. L'article de Morgane Iserte analyse les différentes procédures de contrôle policier de la descente de l'avion jusqu'au renvoi, ainsi que les mesures sécuritaires appliquées à l'aéroport de Roissy par la Police aux frontières et les acteurs privés (Aéroports de Paris et transporteurs) avec la mise en place de technologies d'identification, de surveillance et de traçabilité. A partir d'une observation de la zone d'attente de Roissy, Chowra Makaremi explore les techniques et les effets d'une pénalisation de la circulation qui, au-delà de l'enfermement des étrangers, opère plus largement par une recension et une catégorisation organisant leur mise à l'écart au long cours. La contribution de Christel Cournil fournit, à travers une approche juridique, une analyse de la position du juge de la $\mathrm{CEDH}$ sur la détention et le refoulement des étrangers. Les deux dernières contributions s'intéressent à la gestion de l'asile et aux évolutions du statut de réfugié. L'article de Sarah S. Willen permet d'approcher le traitement des demandeurs d'asile soudanais en Israël en étudiant l'analogie entre le génocide au Darfour et l'Holocauste juif qui conduit à la mise en place d'une logique d'intervention humanitaire d'exception. Finalement, le dilemme de la « sortie » des déboutés des centres d'accueil pour demandeurs d'asile, développé par Carolina Kobelinsky, permet d'analyser le rôle ambigu des intervenants sociaux travaillant pour les associations gestionnaires de l'Etat lorsqu'il s'agit non plus seulement de contrôler mais aussi d'expulser les étrangers.

7. Foucault M., “L’asile illimité”, Dits et Ecrits II, Paris, Gallimard, coll. « Quarto », 2001, p. 275

8. Pandolfi M., 2002, «'Moral entrepreneurs', souverainetés mouvantes et barbelés. Le bio-politique dans les Balkans postcommunistes ", Anthropologie et Sociétés, numéro spécial ; Pandolfi M., Abélès M. (dirs.), Politiques jeux d'espaces, vol. 26, n¹, p. 39. 